\title{
Thermal mismatch stress in SiC whisker reinforced aluminium composite: new measurement method by $\mathbf{X}$-ray diffraction
}

\author{
W. D. Fei, Q. Y. Liu, N. G. Liang, and C. K. Yao
}

A new X-ray diffraction method for characterising thermal mismatch stress (TMS) in $\mathrm{SiC}_{\mathrm{w}}-\mathrm{Al}$ composite has been developed. The TMS and thermal mismatch strain (TMSN) in SiC whiskers are considered to be axis symmetrical, and can be calculated by measuring the lattice distortion of the whiskers. Not only the average TMS in whiskers and matrix can be obtained, but the TMS components along longitudinal and radial directions in the SiC whiskers can also be deduced. Experimental results indicate that the TMS in SiC whiskers is compressive, and tensile in the aluminium matrix. The TMS and TMSN components along the longitudinal direction in the SiC whiskers are greater than those along the radial direction for a $\mathrm{SiC}_{\mathrm{w}}-\mathrm{Al}$ composite quenched at $500{ }^{\circ} \mathrm{C}$.

MST/4586

Professors Fei and Yao are in the School of Materials Science and Engineering, Harbin Institute of Technology, Harbin 150001, China (wdfei@hope.hit.edu.cn) and Dr Liu and Professor Liang are in the State Key Laboratory of Non-linear Mechanics, Institute of Mechanics, Chinese Academy of Sciences, Beijing 100080, China. Manuscript received 11 January 2000; accepted 10 October 2000

(C) 2001 IoM Communications Ltd.

\section{Introduction}

When a metal matrix composite (MMC) is cooled down to room temperature from the fabrication or annealing temperature, thermal residual stresses such as traditional macrostress, microstress, and thermal mismatch stress (TMS) can be induced in the composite. Thermal mismatch stress is generated by the great difference between the coefficients of thermal expansion of the matrix metal and the reinforcement, and has obvious effects on the properties of composites, such as yield strength, fatigue life, dimension stability, etc. ${ }^{1-3}$ For example, the tensile yield strength of $\mathrm{SiC}_{\mathrm{w}}-\mathrm{Al}$ composite is lower than the compressive one, this asymmetric response is a direct consequence of the average tensile residual TMS in the matrix. ${ }^{3}$ In the last decade considerable efforts, both theoretical ${ }^{4-6}$ and experimental, ${ }^{7-9}$ have been made to understand TMS and its effects on the properties of composites.

For example, the model based on Eshelby's equivalent inclusion method has been used to solve the problem of TMS. ${ }^{4}$ The advantages of the Eshelby model are that it can solve three-dimensional composite systems and also can take into account the effects of the fibre volume fraction easily. At the same time, finite element TMS computations have also been performed by Povirk et al. ${ }^{5}$ and Durodola and Derby. ${ }^{6}$ The distribution of TMS in matrix and reinforcement can be estimated. However, for computational purposes, the MMC was first idealised as uniform sized inclusions in a periodic array so that the Eshelby model or the finite element method could be used.

In fact, TMS in random short fibre composite systems is more complicated than that in aligned systems. Unfortunately, less attention was paid to TMS in this kind of system.

Experimentally, $\sin ^{2} \psi$ X-ray diffraction (XRD) and neutron diffraction methods are often used to measure TMS in MMCs. ${ }^{7,8}$ The measurement principles for both of them are the same, but the penetration of neutrons is higher than that of X-rays. ${ }^{8}$ Only the average macrostress and TMS at the surface can be measured by the $\sin ^{2} \psi$ XRD method because both stresses can cause diffraction peak shifts. It is very difficult to distinguish the individual effects of traditional macrostress or TMS on diffraction peak shift; the $\sin ^{2} \psi$ XRD method is unable to resolve TMS. The neutron diffraction method can overcome this disadvantage since the bulk average TMS can be determined. This is because neutrons take advantage of large sampling volumes, so the bulk average macrostress is zero. However, the neutron diffraction method cannot be used widely owing to the availability of neutron sources. Synchrotron $\mathrm{X}$-ray energy dispersive diffraction ${ }^{9}$ is also employed to measure TMS in MMC, which offers the promise of both good penetration and a potentially high spatial resolution of residual strain gradients. Thermal mismatch stress can be measured easily, but the cost of this method is too expensive to be used widely.

A new experimental method is developed in this paper to characterise TMS by measuring the lattice distortion of $\mathrm{SiC}$ whiskers in $\mathrm{SiC}_{\mathrm{w}}-\mathrm{Al}$ composites in which the whiskers are distributed randomly.

\section{Experimental details}

The $\mathrm{SiC}_{\mathrm{w}}-\mathrm{Al}$ composite used, with a $\mathrm{SiC}$ volume fraction of 20 vol.- $\%$, was fabricated by the squeeze casting method and the matrix was pure aluminium. The random distribution of $\mathrm{SiC}$ whiskers in the as cast composite was observed by SEM, as shown in Fig. 1. The size of the specimen for XRD measurement was $5 \mathrm{~mm}$ in diameter and $0.5 \mathrm{~mm}$ thick. The specimen was so thin that the macrostress could be neglected. The specimens were annealed at $500^{\circ} \mathrm{C}$ for $2 \mathrm{~h}$, then quenched in water at room temperature before XRD testing.

The XRD test in a step scanning model was carried out using a Philips X'Pert X-ray diffractometer and a step size of $0.02^{\circ}$. The radiation used was $\mathrm{Cu} K_{\alpha}$, the filter was made from $\mathrm{Ni}$, the operation voltage was $50 \mathrm{kV}$, and the current was $25 \mathrm{~mA}$. The double line of $K_{\alpha 1}$ and $K_{\alpha 2}$ was separated by computer.

\section{Theory}

In general, during changes in temperature three kinds of residual stresses are induced in composites. The first one is 


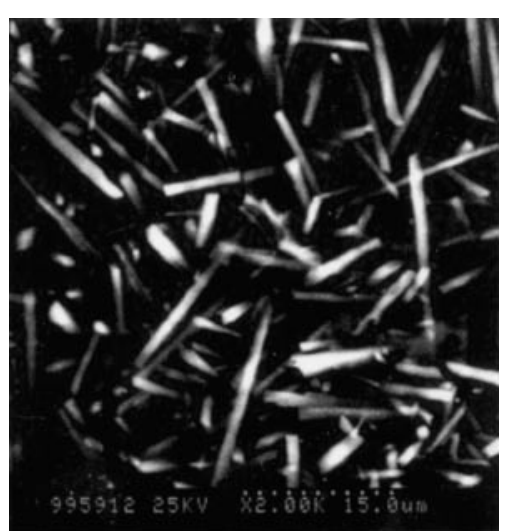

1 Distributions of $\mathrm{SiC}$ whiskers in as cast $\mathrm{SiC}_{\mathrm{w}}-\mathrm{Al}$ composites

macrostress, which is a balance among the different parts of a specimen in the macroscale. The second one is TMS, which keeps balance between the whisker and matrix. The third one is microstress, which stores in defects such as dislocations. In this study, if the specimen is thin enough, the macrostress can be neglected. The microstress only causes a broadening or weakening of XRD peaks. ${ }^{10}$ Therefore, the displacement of the diffraction peaks of whisker and matrix can only be caused by TMS, which is emphasised in this paper.

Thermal mismatch stress is self-equilibrated internal stress because it is free from any other external force or surface constraint. The volume average TMS between the reinforcement and the matrix in $\mathrm{SiC}_{\mathrm{w}}-\mathrm{Al}$ composite is zero, and the signs in them are reverse. So TMS can be known by measuring the displacement of the diffraction peaks of $\mathrm{SiC}$ whiskers or aluminium matrix. A $\mathrm{SiC}$ whisker with high strength can keep pure elastic state when the composite is cooled down from high temperature. Therefore it is more accurate to characterise TMS using the shift in SiC whisker diffraction peaks, since the diffraction angle of the matrix can be affected by many factors such as TMS, solution of alloying elements, stacking fault, and so on. It is difficult to separate the effects on a matrix diffraction peak, which gives less accurate results.

\section{ASSUMPTIONS}

The distribution of whiskers is considered to be random in the composite fabricated by squeeze casting, and the longitudinal directions of the whiskers are random too (as shown in Fig. 1). The whiskers in the volume irradiated by $\mathrm{X}$-ray are also random since there are so many whiskers in them.

It is assumed that the TMS is uniform in a whisker, the difference in TMS at the end and middle region of a whisker is neglected. The difference in TMS among different whiskers is also neglected, because the displacements of diffraction peaks of $\mathrm{SiC}$ whiskers are only related to a mean strain of all whiskers in the volume of XRD. The fluctuation of strain in different whiskers only has a broadening effect on diffraction peaks and no effect on the position of diffraction peaks

The strain and stress in the whisker is assumed to be axially symmetrical, because the aspect ratio of the $\mathrm{SiC}$ whiskers is very large $(\sim 25)$ and the diameter of the whiskers is very small $(\sim 0 \cdot 5-1 \mu \mathrm{m})$. A local column coordinate system for any whisker is shown in Fig. 2. Nonzero stress components are $\sigma_{\mathrm{r}}, \sigma_{\theta}$, and $\sigma_{\mathrm{z}}$, and $\sigma_{\mathrm{r}}=\sigma_{\theta}$ because of the small diameter of the whisker. In this case, the stresses and strains in a whisker can be described by $\sigma_{\mathrm{r}}$ and $\sigma_{\mathrm{z}}$, and $\varepsilon_{\mathrm{r}}$, and $\varepsilon_{\mathrm{z}}$, respectively.

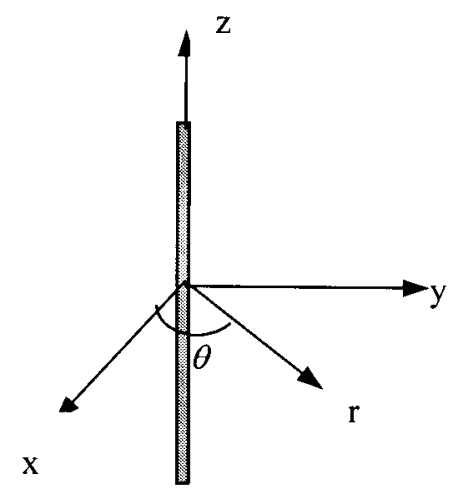

\section{Local column coordinate system fixed in whisker}

\section{STRESS AND STRAIN IN WHISKER}

In a whisker, stresses and strains are related by

$$
\left.\begin{array}{c}
\sigma_{\mathrm{r}}=\lambda \varepsilon+2 \mu \varepsilon_{\mathrm{r}} \\
\sigma_{\mathrm{z}}=\lambda \varepsilon+2 \mu \varepsilon_{\mathrm{z}}
\end{array}\right\}
$$

where $\varepsilon=\varepsilon_{\mathrm{r}}+\varepsilon_{\mathrm{z}}+\varepsilon_{\theta}=\varepsilon_{\mathrm{z}}+2 \varepsilon_{\mathrm{r}}$ is volume strain, $\lambda$ and $\mu$ are Lame constants, $\sigma_{\mathrm{z}}$ and $\sigma_{\mathrm{r}}$ are the longitudinal and radial TMS, respectively. From the above relationship formula, it is obvious that $\sigma_{\mathrm{r}}$ and $\sigma_{\mathrm{z}}$ can be determined by measuring $\varepsilon_{\mathrm{z}}$ and $\varepsilon_{\mathrm{r}}$.

Because of the random and uniform distributions of the whiskers in the composite, the intrinsic phase average stress in the whiskers $\langle\sigma\rangle_{\mathrm{w}}$ is a sphere symmetry tensor

$$
\langle\sigma\rangle_{\mathrm{w}}=\left(\begin{array}{ccc}
\sigma_{\mathrm{w}}^{0} & 0 & 0 \\
0 & \sigma_{\mathrm{w}}^{0} & 0 \\
0 & 0 & \sigma_{\mathrm{w}}^{0}
\end{array}\right)
$$

It is clear that $\langle\sigma\rangle_{\mathrm{w}}$ is the thermal mismatch macrostress. In the macrocoordinate system 123 shown in Fig. 3,

$$
\left\langle\sigma_{33}\right\rangle_{\mathrm{w}}=\sigma_{\mathrm{w}}^{0}
$$

On the other hand, for the whisker of which the longitudinal direction is at a angle of $\phi$ to the 3 axis (see Fig. 3)

$$
\sigma_{33}=\sigma_{\mathrm{z}} \cos ^{2} \phi+\sigma_{\mathrm{r}} \sin ^{2} \phi
$$

Therefore,

$$
\begin{aligned}
\sigma_{\mathrm{w}}^{0} & =\left\langle\sigma_{33}\right\rangle_{\mathrm{w}}=(4 \pi)^{-1} \iint \sigma_{33} \mathrm{~d} \Omega \\
& =(4 \pi)^{-1} \int_{0}^{2 \pi} \int_{0}^{\pi} \sigma_{33} \sin \phi \mathrm{d} \phi \mathrm{d} \theta \\
& =\frac{1}{3} \sigma_{\mathrm{z}}+\frac{2}{3} \sigma_{\mathrm{r}} \quad . \quad . \quad . \quad . \quad .
\end{aligned}
$$

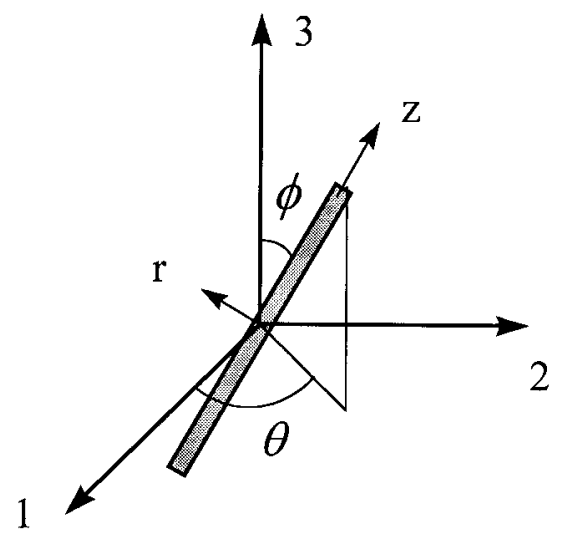

3 Schematic diagram of orientation of SiC whisker corresponding to macrocoordinate 123 
where $\Omega$ is the solid angle. According to above analysis, if the stress components in the whiskers are calculated, the intrinsic phase average TMS can easily be available. Furthermore, according to the balance rule of TMS, intrinsic phase average TMS in the matrix is also obtained in terms of the following equation

$$
V_{\mathrm{f}}\langle\sigma\rangle_{\mathrm{w}}+\left(1-V_{\mathrm{f}}\right)\langle\sigma\rangle_{\mathrm{m}}=0
$$

where $V_{\mathrm{f}}$ is the volume fraction of $\mathrm{SiC}$ whiskers and $\langle\sigma\rangle_{\mathrm{m}}$ is the intrinsic phase average TMS in the matrix, and is a sphere symmetry tensor

$$
\langle\sigma\rangle_{\mathrm{m}}=\left(\begin{array}{ccc}
\sigma_{\mathrm{m}}^{0} & 0 & 0 \\
0 & \sigma_{\mathrm{m}}^{0} & 0 \\
0 & & \sigma_{\mathrm{m}}^{0}
\end{array}\right)
$$

Therefore, equation (4) can be rewritten as follows

$$
V_{\mathrm{t}} \sigma_{\mathrm{w}}^{0}+\left(1-V_{\mathrm{f}}\right) \sigma_{\mathrm{m}}^{0}=0
$$

\section{RELATIONS BETWEEN THERMAL MISMATCH STRAIN AND DIFFRACTION ANGLES IN SiC WHISKER}

Previous studies have indicated that the $\beta$-SiC whisker is a single crystal whose longitudinal direction is $\langle 111\rangle .{ }^{11}$ Only the special crystallographic characteristics of the whisker makes it possible to measure thermal mismatch strain (TMSN) by XRD method (elastic strain). To simplify the analysis, the longitudinal direction of the whisker is denoted by $[111]_{\mathrm{z}}$ as the $z$ axis in the coordinate system in Fig. 4, and the corresponding plane as $(111)_{\mathrm{z}}$. The other $\langle 111\rangle$ direction, being at a angle of $\phi= \pm \cos ^{-1}(1 / 3)$ to the $z$ axis, is denoted as $[111]_{\phi 1}$ and the corresponding plane as $(111)_{\phi 1}$ (see Fig. $4 a$ )

In a random whisker composite system, where the diffraction probability of any plane is not zero, the 111 diffraction peak of the $\mathrm{SiC}$ whisker can be considered as the sum of the $(111)_{z}$ and the $(111)_{\phi 1}$ plane. According to the Rietveld ${ }^{12}$ method, the intensity of the 111 diffraction peak $I_{111}$ can be described as

$$
I_{111}(\theta)=I_{111_{z}} G_{111_{z}}\left(\theta-\theta_{111_{z}}\right)+I_{111_{\phi 1}} G_{111_{\phi 1}}\left(\theta-\theta_{111_{\phi 1}}\right)
$$

where $I_{111_{z}}$ and $I_{111_{1}}$ represent the $(111)_{\mathrm{z}}$ and $(11)_{\phi 1}$ planes, respectively. The integral intensity is $I, G$ is the normalised peak shape function, and $\theta$ is the Bragg angle. The integral intensities contain structure factor, angle factor, temperature factor, and multiplicity factor, and so on. It is assumed that all of above factors are the same for the $(111)_{z}$ and $(111)_{\phi 1}$ planes except for the multiplicity factor. The multiplicity factor of the (111) $)_{\phi 1}$ plane is three times more than that of the $(111)_{z}$ plane, so that equation (9) can be rewritten as

$$
I_{111}(\theta)=c\left[G_{111_{z}}\left(\theta-\theta_{111_{z}}\right)+3 G_{111_{\phi 1}}\left(\theta-\theta_{111_{\phi 1}}\right)\right]
$$

where $c$ is a constant related to structure, angle, and temperature factors. The peak position of the 111 diffraction can be calculated by

$$
\left.\left(\frac{\partial G_{111_{\mathrm{z}}}\left(\theta-\theta_{111_{\mathrm{z}}}\right)}{\partial \theta}+3 \frac{\partial G_{111_{\phi 1}}\left(\theta-\theta_{111_{\phi 1}}\right)}{\partial \theta}\right)\right|_{\theta=\theta_{111}}=0
$$

where $\theta_{111}$ is the peak position angle of the 111 diffraction of the whisker. The normalised peak shape function is selected as the Gauss function, ${ }^{*}$ i.e.

$$
G_{\mathrm{i}}(\theta)=\frac{2(\ln 2)^{1 / 2}}{\left(\pi H_{\mathrm{i}}\right)^{1 / 2}} \exp \left[-\frac{4 \ln 2}{H_{\mathrm{i}}^{2}}\left(2 \theta-2 \theta_{\mathrm{i}}\right)^{2}\right]
$$

\footnotetext{
* It can be proved that the analysis of the present study is correct for any bell shaped function selected as the peak shape function, because only the intensity near the diffraction peak is considered and the thermal mismatch strain is small.
}

where subscript $i$ represents the $(111)_{z}$ or $(111)_{\phi 1}$ plane and $H_{\mathrm{i}}$ is the width at half the intensity of the diffraction peak. Substituting equation (12) into equation (11), and using the Taylor formula, we obtain equation (13) if the high order small quantity is neglected.

$$
\left(\theta_{111}-\theta_{111_{z}}\right)+3\left(\frac{H_{111_{z}}}{H_{111_{\phi 1}}}\right)^{3}\left(\theta_{111}-\theta_{111_{\phi 1}}\right)=0
$$

If the whisker is considered to be a cylinder, the Toraya ${ }^{12}$ method can be employed to analyse the width of the diffraction peak. If the diameter of the whisker is $D$ and the length is $h$ then

$$
H_{\mathrm{i}}=c_{\mathrm{i}} \beta
$$

where $c_{\mathrm{i}}$ is a constant relating to peak shape function and

$$
\left.\begin{array}{l}
\beta=\frac{\lambda}{h \cos \theta} \quad \varphi=0 \\
\beta=\frac{\pi \sin \varphi}{D}\left[\frac{8}{3}+2 q \cos ^{-1} q-\frac{\sin ^{-1} q}{2 q}-\right. \\
\left.\frac{5}{2}\left(1-q^{2}\right)^{1 / 2}+\frac{1}{3}\left(1-q^{2}\right)^{3 / 2}\right]^{-1} \frac{\lambda}{\cos \theta} \quad 0<\varphi \leqslant \chi \\
\beta=\frac{\sin \varphi}{D}\left(\frac{8}{3}-\frac{\pi D}{4 h} \cot \varphi\right)^{-1} \frac{\lambda}{\cos \theta} \quad \chi<\varphi \leqslant \pi / 2
\end{array}\right\}
$$

where $\theta$ is the Bragg angle, $\varphi$ is the angle between the cylinder axis and the diffraction vector, $\chi=\tan ^{-1}(D / h)$, and $q=(h / D) \tan \chi$. Observation by SEM shows that the aspect ratio $h / D$ of the $\beta$-SiC whiskers used is $\sim 25$. For the $(111)_{z}$ diffraction $\varphi=0$ and

$$
\beta_{111_{\mathrm{z}}}=\frac{\lambda}{h \cos \theta}
$$

For $(111)_{\phi 1}$ diffraction $\varphi=70 \cdot 5^{\circ}, \chi=\tan ^{-1}(D / h) \approx 2 \cdot 3^{\circ}$, and $\chi<\varphi<\pi / 2$. Therefore,

$$
\beta_{111_{\phi 1}}=\frac{\sin \varphi}{D}\left(\frac{8}{3}-\frac{\pi D}{4 h} \cot \varphi\right)^{-1} \frac{\lambda}{\cos \theta} .
$$

and

$$
\frac{H_{111_{z}}}{H_{111_{\phi 1}}}=\frac{\beta_{111_{z}}}{\beta_{111_{\phi 1}}}=\frac{D}{h} \sin \phi\left(\frac{8}{3}-\frac{\pi D}{4 h} \cot \phi\right)
$$

Simple calculation indicates that $\left(H_{111_{2}} / H_{111_{\phi_{1}}}\right)^{3} \approx 10^{-3}$, that is to say, the second terms in the left side of equation (13) can be neglected for first approximation. Therefore,

$$
\varepsilon_{111}=\frac{d_{111}-d_{111}^{0}}{d_{111}^{0}}=\varepsilon_{\mathrm{z}}
$$

where $d_{111}$ is the apparent plane spacing determined from the 111 diffraction peak using Bragg's law, $d_{111}^{0}$ is the $\{111\}$ plane spacing of stress free whiskers, and $\varepsilon_{111}$ is the apparent strain defined by equation (19). It can be found that the peak position of the 111 diffraction of the $\mathrm{SiC}$ whiskers is given by the $(111)_{z}$ diffraction. Although the 111 diffraction peak is contributed both by the $(111)_{z}$ and the $(111)_{\phi 1}$ plane, the broadening effect of $(111)_{\phi 1}$ is so great that the position of the 111 diffraction peak is determined by the $(111)_{z}$ diffraction mainly near the top of the 111 diffraction peak.

The normal direction of the $\{200\}$ plane is at an angle of $\phi_{2}= \pm \cos ^{-1} 3^{-1 / 2}$ to the $[111]_{\mathrm{z}}$ direction ( $\mathrm{z}$ direction) as shown in Fig. $4 b$. The strain obtained from the 200 diffraction peak is at the angle of $\phi_{2}$ to the longitudinal direction in the whisker, and

$$
\varepsilon_{200}=\varepsilon_{\phi_{2}}=\cos ^{2} \phi_{2} \varepsilon_{\mathrm{Z}}+\sin ^{2} \phi_{2} \varepsilon_{\mathrm{r}}=\frac{1}{3} \varepsilon_{\mathrm{Z}}+\frac{2}{3} \varepsilon_{\mathrm{r}}
$$

where the subscript 200 represents the $\{200\}$ plane.

The sum total of $\{220\}$ planes is 12 , which can be divided into two groups. Six planes are perpendicular to the $z$ 


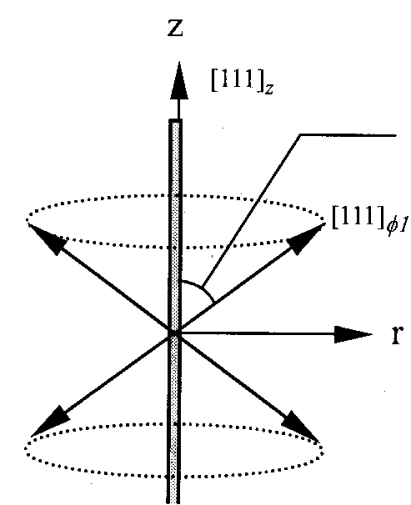

(a)

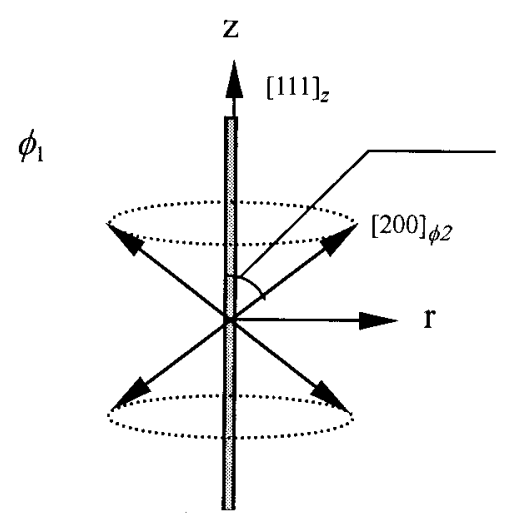

(b)

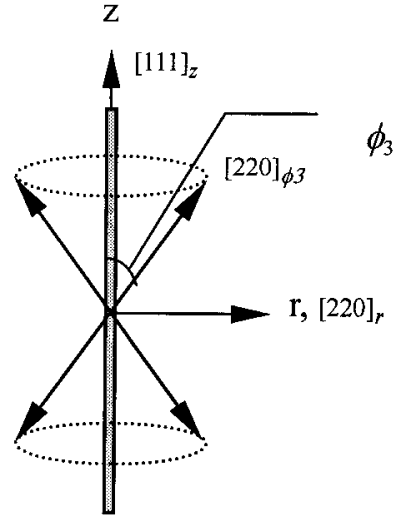

(c)

$a\langle 111\rangle ; b\langle 200\rangle ; c\langle 220\rangle$

\section{Distributions of different directions corresponding to longitudinal direction of SiC whisker}

direction, denoted by $(220)_{z}$. The other 6 planes are at an angle of $\phi_{3}= \pm \cos (2 / 3)^{1 / 2}$ to the $z$ direction of the whisker, denoted by $(220)_{\phi 3}$, as shown in Fig. $4 c$.

Analysis for $\{220\}$ is similar to that of $\{111\}$ diffraction

$$
\left(\theta_{220}-\theta_{220_{\mathrm{r}}}\right)+\left(\frac{H_{220_{\mathrm{r}}}}{H_{220_{\phi 3}}}\right)\left(\theta_{220}-\theta_{220_{\phi 3}}\right)=0
$$

The multiplicity factor of $(220)_{\mathrm{r}}$ and $(220)_{\phi 3}$ are the same, so there is no constant factor 3 before the second term in the left side of equation (21) compared with that of equation (13). If the aspect ratio of the whisker is taken as 25 , the calculated result using equation (15) gives

$$
\theta_{220}=\frac{5}{6} \theta_{220_{\phi 3}}+\frac{1}{6} \theta_{220_{\mathrm{r}}}
$$

Differentiating both sides of equation (22) and combining it with Bragg's equation give

$$
\varepsilon_{220}=\frac{5}{6} \varepsilon_{220_{\phi 3}}+\frac{1}{6} \varepsilon_{220_{\mathrm{r}}}
$$

Similarly,

$$
\left.\begin{array}{l}
\varepsilon_{220_{\phi 3}}=\sigma_{\mathrm{z}} \cos ^{2} \phi_{3}+\sigma_{\mathrm{r}} \sin ^{2} \phi_{3} \\
\varepsilon_{220_{\mathrm{r}}}=\varepsilon_{\mathrm{r}}
\end{array}\right\}
$$

Therefore, equation (23) can be rewritten as

$$
\varepsilon_{220}=\frac{5}{9} \varepsilon_{\mathrm{z}}+\frac{4}{9} \varepsilon_{\mathrm{r}}
$$

In principle $\varepsilon_{\mathrm{z}}, \varepsilon_{200}$, and $\varepsilon_{220}$ can be measured by XRD, so that the strain components in the whiskers can then be calculated. Using equation (1), the stress components in the whiskers can be obtained and the intrinsic phase average TMS in the matrix can also be deduced by equation (8).

\section{Measurement method and results}

\section{MEASUREMENT METHOD}

According to the above analysis, we know that the intrinsic phase average TMS in the $\mathrm{SiC}$ whiskers can be calculated by measuring the peak positions of diffraction peaks such as 111,200 , and 220 . The precision of calculation strongly depends on the measuring precision. In general, a high angle diffraction may be measured more precisely than a low one, but there are two reasons why high angle diffraction peaks are not suitable for the present study. First, the relationships between the stress components and the displacements are very complicated. Second, the volume fraction of whiskers in the whisker-Al composite is about $0 \cdot 2-0.3$ or less. The intensities of the diffraction peaks are very weak, which can result in an uneven effect on the diffraction peaks and a low precision in determining diffraction peak position. So, 111,200 , and 220 diffractions are recommended.

Because the diffraction angles of 111, 200, and 220 diffraction are low, normal scanning measuring technique may bring errors. Therefore, the step scanning method is employed, in which the step size is $0.02^{\circ}$ and the sampling time is $20 \mathrm{~s}$ per step. As is well known, an error in the diffraction angle is mainly caused by an error in the initial angle of step scanning. For this reason, we propose that the difference between two diffraction angles can be used instead of the angle of one diffraction peak to determine the strain in the $\mathrm{SiC}$ whiskers.

Using differentiation of Bragg's equation, the following equations can be obtained for 220 and 200 diffractions.

$$
\begin{aligned}
\delta_{1} & =\left(\theta_{220}-\theta_{200}\right)-\left(\theta_{220}^{0}-\theta_{200}^{0}\right) \\
& =\left(\theta_{220}-\theta_{220}^{0}\right)-\left(\theta_{200}^{0}-\theta_{200}\right) \\
& =\tan \theta_{200}^{0} \varepsilon_{200}-\tan \theta_{220}^{0} \varepsilon_{220}
\end{aligned}
$$

where $\theta_{200}^{0}$ and $\theta_{220}^{0}$ are the 200 and 220 diffraction angles of stress free $\mathrm{SiC}$ whiskers, which can be determined by the diffraction of pure $\mathrm{SiC}$ whiskers in the same diffraction conditions.

Similar relation is used for 220 and 111 diffraction

$$
\begin{aligned}
\delta_{2} & =\left(\theta_{220}-\theta_{111}\right)-\left(\theta_{220}^{0}-\theta_{111}^{0}\right) \\
& =\tan \theta_{111}^{0} \varepsilon_{111}-\tan \theta_{220}^{0} \varepsilon_{220}
\end{aligned}
$$

where $\theta_{111}^{0}$ is the 111 diffraction angle of stress free $\mathrm{SiC}$ whiskers.

Combining equations (19), (20), (25), (26), and (27), gives

$$
\begin{aligned}
& \varepsilon_{\mathrm{Z}}=\frac{6 a\left(\delta_{1}-\delta_{2}\right)+9 \delta_{2} a}{9 c a-3 a b-6 b c} \\
& \varepsilon_{\mathrm{r}}=\frac{(9 c-5 b)\left[6 b\left(\delta_{1}-\delta_{2}\right)+9 a \delta_{2}\right]}{4 b(9 a c-3 a b-6 b c)}-\frac{9 \delta_{2}}{4 b}
\end{aligned}
$$

where $a=\tan \theta_{200}^{0}, b=\tan \theta_{200}^{0}$, and $c=\tan \theta_{111}^{0}$

It should be noted that the differences among the angles of 111,200 , and 220 diffraction peaks are used instead of the angles of 111,200 , and 220 diffraction peaks to calculate strains in whiskers so that the error in the initial angle can be reduced greatly. Combining equations (28) and (1), the stress components can be obtained. The average TMS in $\mathrm{SiC}$ whiskers can be calculated by equation (5) and in the matrix by equation (8). 
Table 1 Thermal mismatch strain and thermal mismatch stress in SiC whiskers

\begin{tabular}{lllll}
\hline \multicolumn{2}{l}{ Strain, $\times 10^{-3}$} & & \multicolumn{2}{l}{ Stress, MPa } \\
\cline { 1 - 1 } Radial & Longitudinal & & Radial & Longitudinal \\
\hline$-1 \cdot 7$ & $-2 \cdot 0$ & & -1080 & -1180 \\
\hline
\end{tabular}

\section{RESULTS AND DISCUSSION}

The TMSN and TMS in SiC whiskers are shown in Table 1. The TMSN in Table 1 is the average of three measurements. Along the longitudinal direction, TMSN and TMS are greater than those along the radial direction in the $\mathrm{SiC}$ whiskers.

In general, only average TMS can be deduced by X-ray or neutron diffraction. However, in the present study, both average TMS and the stress components in $\mathrm{SiC}$ whiskers could be obtained. The difference between the longitudinal and radial stress components is because of the difference in stress relaxation along the longitudinal and radius directions in $\mathrm{SiC}$ whiskers.

The intrinsic phase average TMS in the whiskers and the matrix was -1110 and 245 , respectively. It is found that the TMS in the $\mathrm{SiC}$ whiskers is compressive, and is tensile in matrix in $\mathrm{SiC}_{\mathrm{w}}-\mathrm{Al}$ composite. These results agree well with previous studies. ${ }^{13,14}$

It must be pointed out that the measured error for diffraction angles is $\sim 0.01^{\circ}$ in this study, the corresponding fluctuation in the strain in the $\mathrm{SiC}$ whiskers is $\sim 0.0001 \%$ and the fluctuation in stress is $15 \mathrm{Mpa}$. Therefore, it can be concluded that the measured error has no influence on the distribution rule of TMS in SiC whiskers. The TMS in the composite with any heat treatment condition can be conveniently measured. While, if the TMS is calculated by the diffraction of the matrix in an aluminium matrix composite, a stress free aluminium alloy must be prepared. Because precipitation in the composite is quite different from that in an unreinforced aluminium alloy, it is difficult to obtain stress free aluminium with the same state in the aluminium matrix in the composite, which must result in low measurement accuracy.

\section{Conclusions}

1. A new X-ray diffraction method has been developed to characterise thermal mismatch stress (TMS) in a $\mathrm{SiC}_{\mathrm{w}}-\mathrm{Al}$ composite. Both the stress components in $\mathrm{SiC}$ whiskers and the average TMS in $\mathrm{SiC}$ whiskers and the aluminium matrix can be obtained.

2. The TMS and thermal mismatch strain components along the longitudinal direction in $\mathrm{SiC}$ whisker are smaller than along the radial one.

3. The TMS in SiC whiskers was compressive and tensile in the matrix in the as cast $\mathrm{SiC}_{\mathrm{w}}-\mathrm{Al}$ composite.

4. The advantages of this new method are as follows:

(i) The longitudinal and radial components of TMS in $\mathrm{SiC}$ whiskers can be obtained

(ii) The method presented in this paper can be extended to other whisker reinforced metal matrix composites, if the whiskers are single crystal, such as $\mathrm{Si}_{3} \mathrm{~N}_{4}$, $\mathrm{Al}_{2} \mathrm{O}_{3}$, and $\mathrm{Al}_{18} \mathrm{~B}_{4} \mathrm{O}_{33}$ whiskers. The method can also be employed to measure the TMS of highly textured whisker reinforced metal composites.

\section{Acknowledgements}

This work was supported by National Natural Sciences Foundation of China (grant Nos. 59631080 and 19872065)

\section{References}

1. H. Y. ZHANG, P. M. ANDERSON, and G. S. DAEHA: Metall. Mater. Trans. A, 1994, 25A, 415-425.

2. L. C. DAVIS and J. E. ALliwon: Metall. Mater. Trans. A, 1993, 24A, 2487-2469.

3. R. J. ARSENAUlt and S. B. WU: Mater. Sci. Eng., 1987, 96, $77-81$.

4. C.-H. HSUEH and P. F. BECHE: Fibre. Mater. Sci. Eng. A, 1996, 212, 29-35

5. G. L. POVIRK, M. G. STOUT, and M. BOURKE: Acta Metall. Mater., 1992, 40, 2391-2412.

6. J. F. DURODOlA and B. DeRbY: Acta Metall. Mater., 1994, 42, $1525-1543$.

7. H. M. LEDBETTER and M. W. AUSTIN: Mater. Sci. Eng., 1987, 89, $53-61$.

8. N. SHI, R. J. ARSENAULT, A. D. KRAWITZ, and L. F. SMITH: Metall. Trans. A, 1993, 24A, 187-196.

9. T. A. KUNTZ, H. N. G. WADLEY, and D. R. BLACK: Metall. Trans. $A, 1993,24 A, 1117-1124$.

10. X. FANG: in 'X-ray diffraction in metals', $92-102$; 1989, Beijing, Machinail Industry Process.

11. L. GENG and C. K. YAO: Scr. Metall. Mater., 1995, 33, 949-952.

12. M. LIDUn: Prog. in Phys., 1996, 16, 251-271.

13. MEADA and K. WAKASHIMA: Scr. Mater., 1997, 36, 335-340.

14. N. J. CHUN and I. M. DANIEL: Compos. Eng., 1995, 5, 425-436. 